\title{
The Control of Heat Exchanger Outlet Temperature Based on Smith Predictor and Dynamic Matrix Control
}

\author{
Li Ruifeng ${ }^{1, a}$, Wang Liang ${ }^{2, b}$ \\ ${ }^{1}$ School of Automation Science and Electrical Engineering, Beihang University, Beijing, China \\ ${ }^{2}$ School of Automation Science and Electrical Engineering, Beihang University, Beijing, China \\ ambjkl@sina.com, ${ }^{b}$ wangliang@buaa.edu.cn
}

Keywords: heat exchanger, temperature control, Smith predictor, dynamic matrix control

\begin{abstract}
.
It is extremely significant to precisely control exit temperature of heat exchanger in industrial production. However, traditional control strategy has a great deficiency to be applied to temperature control system which is nonlinear, large inertia, pure delay and variant parameters. Smith predictor (SP) provides an effective method to compensate for time delays, but it might make the system unstable out of mismatch of identification model. Dynamic matrix control (DMC) can improve system performance by multiple steps prediction and online rolling optimization on the basis of feedback correction. This paper compares and analyzes the advantages and disadvantages of the above two algorithms by the means of modeling and simulation.
\end{abstract}

\section{Introduction}

Heat exchanger is widely used in many industrial situations to exchange or transmit heat between hot and cold liquid. Control of its outlet temperature is a complex process due to its low response and non-linear behavior caused by many factors such as leakage, friction, etc [1]. Conventional PID controller combined with other intelligent algorithm is generally applied to such plants to obtain satisfactory results when the pure time delay is negligible or small. But the system will become unacceptable when dead time is too large regardless of tuning of the primary controller [2].

A valid method to eliminate the influence of response lag is to adapt Smith predictor to acquire feedback information in advance, which can optimize control strategy ahead of time according to setpoint and predicted output [3]. Though Smith predictor (SP) offers potential improvement in the closed loop performance over traditonal controllers, it is faced with a serious problem of inevitable mismatches between model and actual process [4].

Model Predictive Control (MPC) is another feasible scheme to forecast multiple model response by constructing prediction model, which contains many advanced approaches such as DMC [5]. Dynamic Matrix Control (DMC) uses step response representation to predict the input and output.

The paper is organized as follows. The structure of SP is formulated in the first section, which compares the performance of classical SP with advanced SP. Next part discusses the principle of DMC, which introduces calculation flow of DMC algorithm. Third section tests the characteristics of the two control strategies aimed at the same plant on the condition with huge time delay and inexact model in the platform installed with MATLAB. Finally, conclusions are given in section 4.

\section{Structure of Smith Predictor (SP).}

Classical SP. The structure of classical Smith Predictor is shown in Fig. 1, whers Gc(s) is the controller, Gp(s) is the actual plant, Gm(s) is the model, and Gmo(s) is the delay free part of $\mathrm{Gm}(\mathrm{s})$. The closed loop transfer function between the reference input $r(s)$ and the output $y(s)$ is

$$
G_{1}(s)=\frac{\left.G_{c}(s) G_{p}(s)\right)}{1+G_{c}(s)\left(G_{m o}(s)-G_{m}(s)+G_{p}(s)\right)} .
$$


when model is perfectly equal to actual plant, i.e. $G_{p}(s)=G_{m}(s)$, the characteristic equation is given by (Eq. 2)

$$
1+G_{c}(s) G_{m o}(s)=0 .
$$

This implies that the characteristic equation is free of the time delay so that the primary controller $\mathrm{Gc}(\mathrm{s})$ can be designed with respect to $\mathrm{Gm}(\mathrm{s})$. The achievable performance can thus be greatly improved over a conventional system without delay compensation.

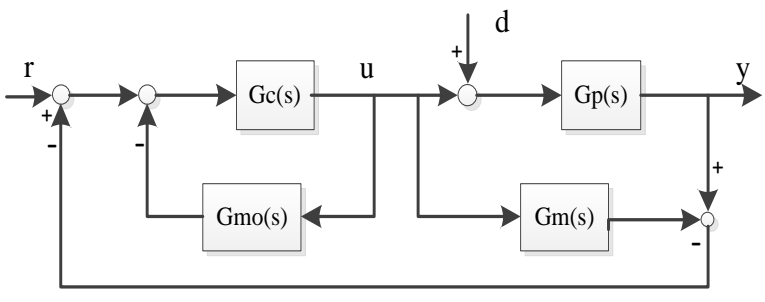

Fig. 1 Structure of Classical Smith Predictor

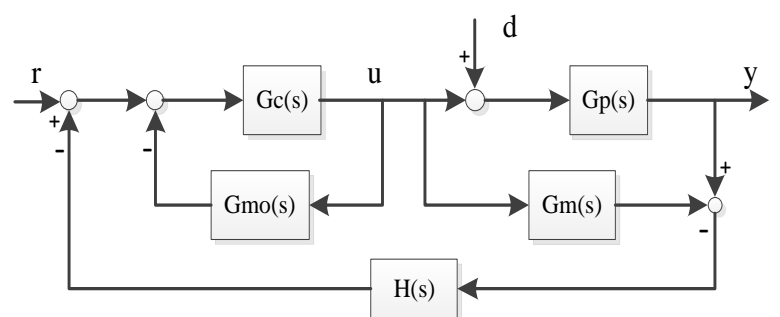

Fig. 2 Structure of Advanced Smith Predictor

Advanced SP. Fig. 2 illustrates the structure of advanced Smith Predictor (SP), where H(s) is creatively added feedback part that is a typical low order low pass filter. The design of $\mathrm{H}(\mathrm{s})$ can strengthen the anti-jamming ability of the system. The closed loop transfer function from the reference input $\mathrm{r}(\mathrm{s})$ to the output $\mathrm{y}(\mathrm{s})$ is

$$
G_{r}(s)=\frac{G_{c}(s) G_{p}(s)}{1+G_{c}(s) G_{m o}(s)+G_{c}(s) H(s)\left(G_{p}(s)-G_{m}(s)\right)} .
$$

The closed loop transfer function between the disturb d(s) and the output $\mathrm{y}(\mathrm{s})$ is

$$
G_{d}(s)=\frac{G_{p}(s)\left(1+G_{c}(s) G_{m o}(s)+H(s) G_{c}(s) G_{m}(s)\right)}{1+G_{c}(s) G_{m o}(s)+G_{c}(s) H(s)\left(G_{p}(s)-G_{m}(s)\right)} .
$$

Eq. 4 can evaluate the robustness of system when choosing different filter. This paper will employ one order low pass filter as feedback unit.

Principle of Dynamic Matrix Control (DMC). DMC is a model predictive control method based on the step response model, which includes mainly the following three parts: prediction model, rolling optimization and feedback correction. Fig. 3 is a detailed control block diagram describing DMC.

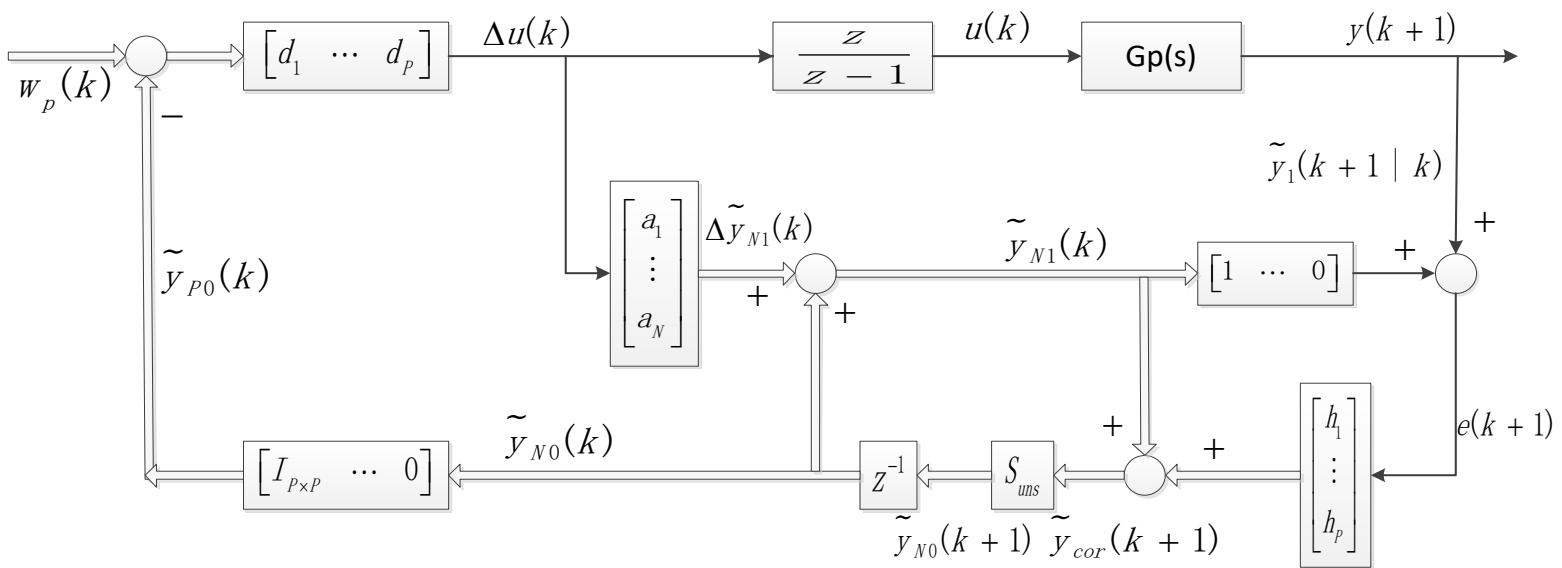

Fig. 3 Control Block Diagram of DMC 
Predictive Model. Prediction model makes full use of coefficient information of step response to get coming equivalent model output state.

The coefficients of the system response under step input signal are $a_{i}=a(i \mathrm{~T})$, where $i=1,2, \cdots$, and $\mathrm{T}$ is the sampling period. When the plant reaches equilibrium, there exists $a_{i} \approx a_{N}(i>\mathrm{N})$, so the system dynamic information can be described approximately by the vector $a=\left[\begin{array}{lll}a_{1} & \cdots & a_{N}\end{array}\right]^{T}$ where $\mathrm{N}$ is modeling domain. At time $(\mathrm{k})$, suppose that the control input remains unchanged, and the future $\mathrm{N}$ predictive outputs are $\widetilde{\mathrm{y}}_{0}(k+i \mid k)$. Giving $\mathrm{M}$ increments $\Delta \mathrm{u}(k), \cdots, \Delta(k+M-1)$, the future outputs $\widetilde{\mathrm{y}}_{M}(k+i \mid k)$ at time $(k+i)$ is

$$
\tilde{y}_{M}(k+i \mid k)=\tilde{y}_{0}(k+i \mid k)+\sum_{j=1}^{\min (M, i)} a_{i-j+1} \Delta u(k+j-1), i \in[1, N] .
$$

Rolling Optimization. Rolling optimization calculates most suitable controller output so as to minimize difference between predicted values and reference points in limited time domain.

The cost function adopted in DMC as follows

$$
\begin{aligned}
& \text { Min } J(k)_{\Delta u_{M}(k)}=\left\|w_{p}(k)-\widetilde{\mathrm{y}}_{P M}(k)\right\|_{Q}^{2}+\left\|\Delta u_{M}(k)\right\|_{R}^{2} \cdot \\
& \text { where } \quad \tilde{\mathrm{y}}_{P M}(k)=\widetilde{\mathrm{y}}_{P 0}(k)+A \Delta u(k), \quad w_{p}(k)=[w(k) \quad \cdots \quad w(k+P)]^{T} \\
& \tilde{\mathrm{y}}_{P 0}(k)=\left[\begin{array}{c}
\tilde{y}_{0}(k+1 \mid k) \\
\vdots \\
\tilde{y}_{0}(k+P \mid k)
\end{array}\right], \quad \tilde{\mathrm{y}}_{P M}(k)=\left[\begin{array}{c}
\tilde{y}_{M}(k+1 \mid k) \\
\vdots \\
\tilde{y}_{M}(k+P \mid k)
\end{array}\right], \quad A=\left[\begin{array}{ccc}
a_{1} & \cdots & 0 \\
\vdots & \ddots & \vdots \\
a_{M} & \cdots & a_{1} \\
\vdots & \ddots & \vdots \\
a_{P} & \cdots & a_{P-M+1}
\end{array}\right] .
\end{aligned}
$$

$A$ is dynamic matrix; $\mathrm{w}_{\mathrm{p}}(\mathrm{k})$ is the desired multiple outputs; $Q$ and $R$ are weighting matrices; $M$ is control domain; $P$ is optimization domain. Optimal minization calculated by (Eq. 6) is

$$
\Delta u_{M}(k)=(A Q A+R)^{-1} A^{T} Q\left[w_{p}(k)-\widetilde{y}_{P 0}(k)\right] .
$$

$M$ control increments $\Delta u_{M}(k)$ are solved by (Eq. 8) at one period, but the implemented is only the first value $\Delta u(k)$. Next time point, $\Delta u(k+1)$ is recalculated as output of the controller.

Feedback Correction. Feedback correction can overcome the interference brought by the inaccuracy of the system model.

At time $(k)$, the control move $\Delta u(k)$ is implemented and the output prediction is

$$
\tilde{y}_{N 1}(k)=\tilde{y}_{N 0}(k)+a \Delta u(k) .
$$

$\tilde{y}_{N 1}(k)$ is the output prediction when the control moves at time $(k+1)$ and keeps invariant in the future time instants, so it is the basis for constructing initial output prediction after shifting $\tilde{y}_{N 1}(k)$ next time. In order to overcome the effects of model uncertainties and interference, at time $(k+1)$, the system output error is denoted as $\mathrm{e}(k+1)=\mathrm{y}(k+1)-\tilde{y}_{1}(k+1 \mid k)$, which is used for feedback correction, and the modified future output prediction is described by

$$
\tilde{y}_{c o r}(k+1)=\tilde{y}_{N 1}(k)+h e(k+1) \text {. }
$$

where $h=\left[\begin{array}{lll}h_{1} & \cdots & h_{N}\end{array}\right]^{T}$ is correction vector.

As time changes, $\tilde{y}_{c o r}(k+1)$ should be shifted to be the initial predictive value of time $(k+1)$ denote 


$$
\tilde{y}_{0}(k+1+i \mid k+1)=\tilde{y}_{c o r}(k+1+i \mid k+1), i \in[1, N-1] .
$$

For a stable system, $\tilde{y}_{0}(k+1+N \mid k+1)$ equals $\tilde{y}_{0}(k+N \mid k+1)$ by approximation, and the shifting process can be represented as follows

$$
\tilde{y}_{N 0}(k+1) \approx \mathrm{S}_{\text {uns }} \tilde{y}_{c o r}(k+1) \text {, where shift matrix } S_{\text {uns }}=\left[\begin{array}{ccccc}
0 & 1 & \cdots & 0 & 0 \\
\vdots & \vdots & \ddots & \vdots & \vdots \\
0 & 0 & \cdots & 0 & 1 \\
0 & 0 & \cdots & 0 & 1
\end{array}\right]
$$

\section{Simulation and Verification.}

In general, the heat exchanger outlet temperature control system can be considered as a first-order plant as (Eq. 13), where $\mathrm{K}_{\mathrm{p}}$ is proportion gain, $T_{p}$ stands for inertia constant, Tol denotes time delay. The distinct of Smith Predictor (SP) algorithm and Dynamic Control Matrix (DMC) algorithm is studied under the condition of disturbance and model mismatch. The input signal is step signal with final value being 1 . The sampling period is set as $10 \mathrm{~s}, \mathrm{~K}_{\mathrm{p}}=1, T_{p}=60, \mathrm{Tol}=40$.

$$
G_{p}(s)=\frac{K_{p}}{1+T_{p} \cdot s} e^{-T o l \cdot s}
$$

Disturbance Rejection. When applying 10\% noise disturbance relative to the set track to the controller output, SP strategy has stronger anti-interference ability and quicker response with rise time being 180s, and DMC algorithm deviates from stable value of the plant with steady error being 0.08 and has lager overshoot about $10 \%$ as shown in Fig. 5.

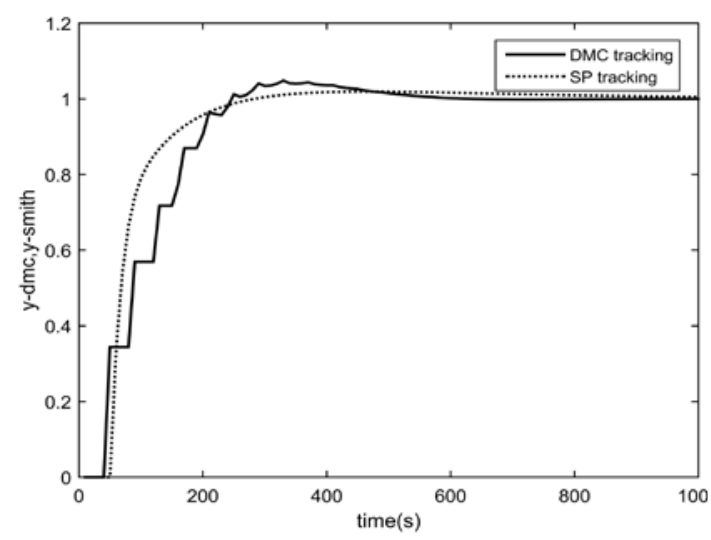

Fig. 4 step response without noise

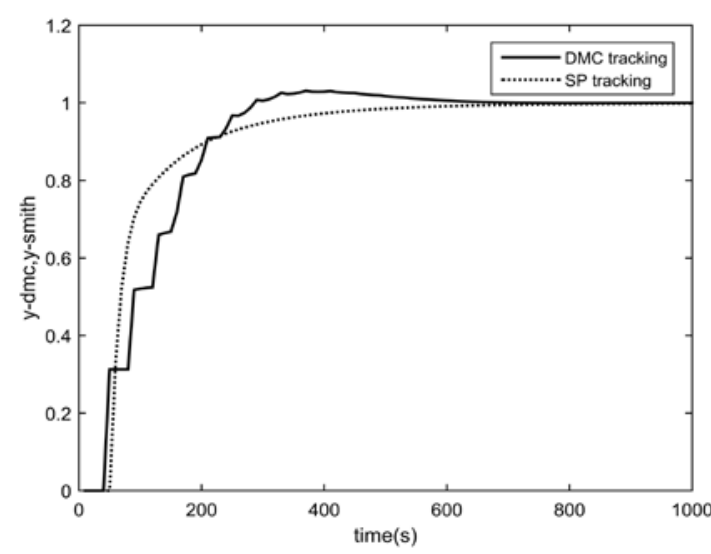

Fig. 6 step response when Kp increases 10\%

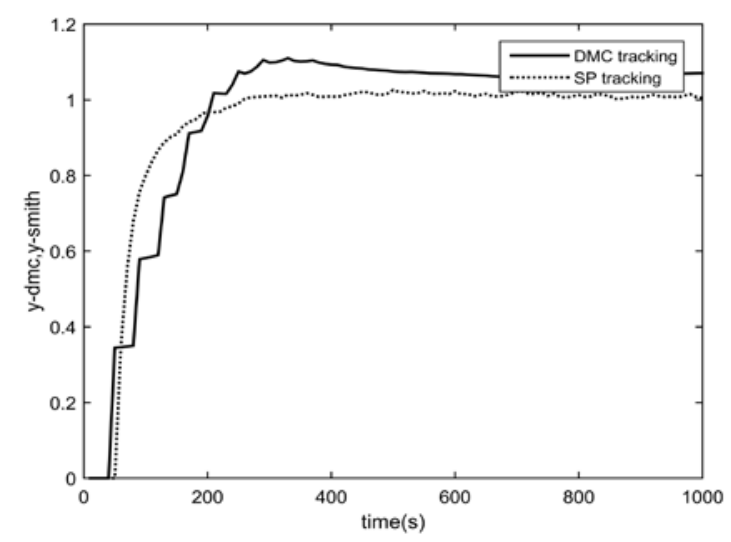

Fig. 5 step response with noise

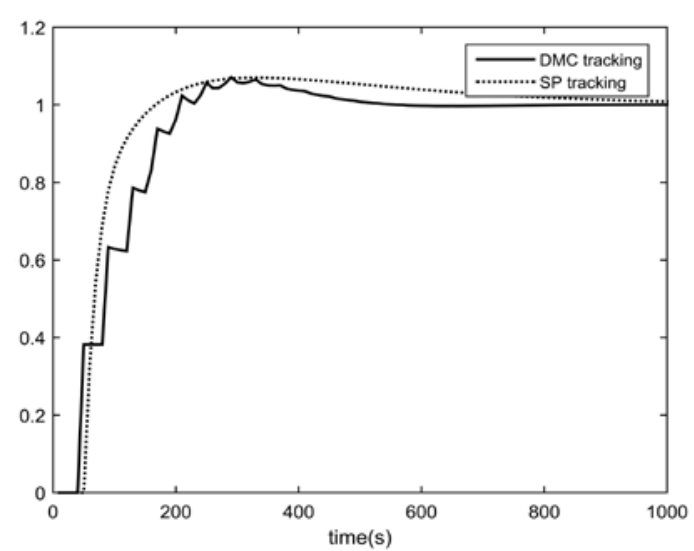

Fig. 7 step response when Kp decreases 10\% 
Model Mismatch. In order to test robustness to variations in process parameters, the simulations are repeated for a 10\% increase or decrease in gain and time delay. The closed-loop responses with parameters floating are described in Fig. 6 to Fig. 9. When gain increases 10\%, step response of DMC has shorter adjustment time approximately $400 \mathrm{~s}$ in the same overshooting of $5 \%$. Whatever the value of time delay is longer or shorter, SP algorithm always exists greater stable error which weakens dynamic performance of the system.

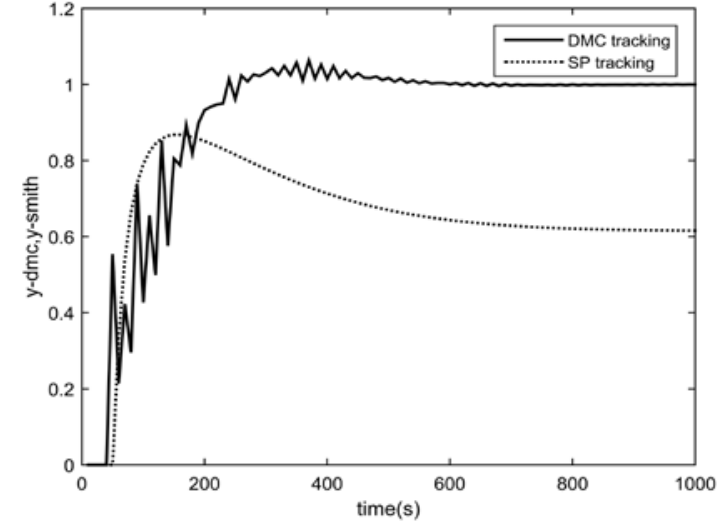

Fig. 8 step response when Tol increases $10 \%$

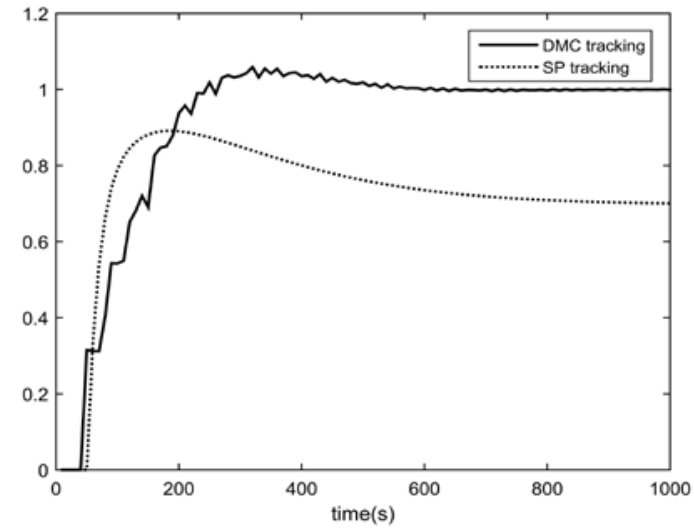

Fig. 9 step response when Tol decreases 10\%

\section{Conclusion}

The result shows that Smith and DMC algorithm are effective to improve performance of the system with time delay with identification model being accurate. However, once parameters of the process are inconsistent with actual plant, SP algorithm has poor performance, especially while time delay mismatches slightly. DMC algorithm provides a feasible solution to suppress the above defects. On the other hand, SP control strategy can reject disturbance of controller output, which is worth further research to restrain noise in DMC algorithm.

\section{Summary}

The paper proposed two control algorithm of heat exchanger outlet temperature, which are Smith Predictor (SP) and Dynamic Matrix Control (DMC). The performance analysis and comparison between the above two blue prints is performed by simulation. And the results suggested that DMC was a better choice when model parameters mismatching with actual system.

\section{References}

[1] Guo D. Research of Heat Exchanger Outlet Temperature and Liquid Level Cascade Control System[J]. Science \& Technology Information, 2010.

[2] Duka A V, Oltean S E. Fuzzy control of a heat exchanger[C]// IEEE International Conference on Automation Quality and Testing Robotics. IEEE, 2012:135-139.

[3] Vrecko D, Vrancić D, Juricić D, et al. A new modified Smith predictor: the concept, design and tuning.[J]. Isa Transactions, 2001, 40(2):111-121.

[4] Zhang W, Xu X. Analytical design and analysis of mismatched Smith predictor.[J]. Isa Transactions, 2001, 40(2):133-138.

[5] Kokate R D, Waghmare L M. IMC-PID and Predictive Controller Design for a Shell and Tube Heat Exchanger[C]// International Conference on Emerging Trends in Engineering and Technology. IEEE Xplore, 2010:1037-1041.

[6] C. B. Robustness analysis of DMC for first order plus dead-time processes[C]// World Congress. 2002:140-140. 